\title{
Research on Control Strategies for Performance Optimization of Wireless Powering System for Smart Home Appliances
}

\author{
Zhenchao Xie*, Daohong Lin and Wangcheng Zhu \\ Electric Power Research Institute of Hainan Power Grid Co., Ltd., Haikou 570125, Hainan Province, China \\ ${ }^{*}$ Corresponding author
}

\begin{abstract}
With the development of smart technology and the improvement of living standard, the demand for a life with smart home appliance is increasing. The advancement of wireless power transfer technology has provided an opportunity to charge home appliance wirelessly. The combination of smart home appliance and wireless power transfer will make life more intelligent. The one-to-many wireless power supply system of smart home appliance and its control strategy optimization is studied. Firstly, the equivalent circuit model of one-to-many wireless power supply system of smart home appliance is set up and the influence of load on the output power and transfer efficiency is analyzed. Afterwards, the control strategy of power is studied, including power stability and emergency power supply. Finally, the experiment platform of one-to-many wireless power supply system is constructed, and the power transfer capacity and load property is tested, verifying the theoretic analysis.
\end{abstract}

Keywords-smart home appliance; wireless power transfer; multiple load; performance Introduction

\section{INTRODUCTION}

With the rapid development of science and technology, people continue to pursue higher quality of life. Therefore, the concept of smart home appliances was born. Smart home appliances have been widely used in nearly every country in the world, and in-depth study on the design of user interface and the collaborative working mechanism among smart home devices have been conducted. But now home appliances are still powered by traditional electricity supply system, which demands connectors to achieve electrical connection. In order to make home appliances more intelligent, the research on wireless power system for smart home appliances attracts more and more attentions. The application of wireless power transmission technology greatly improves the flexibility, convenience and reliability of smart home appliances. It is also of great significance to the further introduction of smart home appliances [1-5].

Wireless electricity transmission technology can be achieved through multiple means, such as electromagnetic induction, magnetic coupling resonance, microwave, etc. The technology is able to meet the demand of the smart home appliances system, with its transmission distance ranging from a few centimeters to several meters, and its transmission power reaching hundreds of kilowatts. As security is a major concern in the real application of the microwave radio transmission technology, wireless power transmission is mostly materialized by electromagnetic induction and magnetic resonance coupling. In order to improve the transmitting efficiency of the system and reduce the sensitivity of the system performance to relevant parameters involved, this paper mainly studies the wireless power supply system for smart home appliances based on the magnetic coupling resonant technology [6-8].

At present, the research on wireless power transmission technology for smart home appliances has become a hot topic in academia and a popular destination for investment in the business world [9]. Great progress has been made in the field in terms of theoretical modeling, system control, resonator design, optimization of overall operating characteristics, and specific applications. However, most research at present still focuses on the "one-to-one" wireless power supply system. Research on the "one to many" system (one power transmitting coil supplies power to multiple loads) is still under exploration. Many key problems are yet to be resolved. There is still a long way to go before this system is put into real application.

\section{ANALYSIS OF THE WIRELESS POWER SUPPLY SYSTEM FOR SMART HOME APPLIANCES}

A. Modeling of the Wireless Power Supply System for Smart Home Appliances

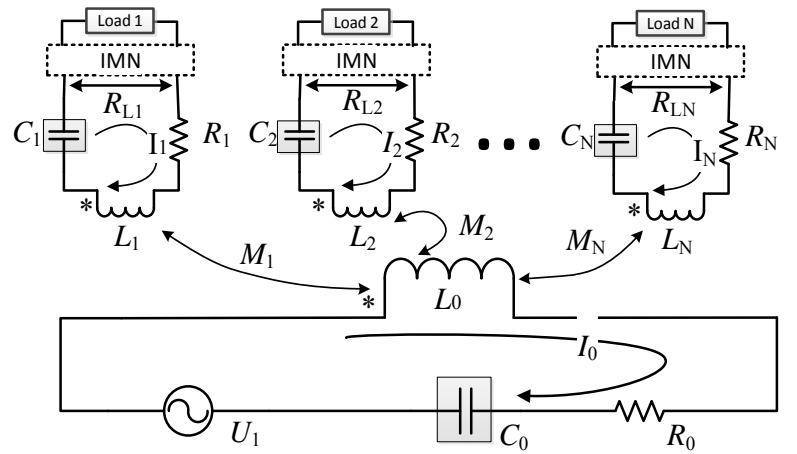

FIGURE I. $\quad$ EQUIVALENT CIRCUIT OF WIRELESS POWER TRANSFER OF SMART HOME APPLIANCES

The wireless power supply system for smart home appliances mainly consists of the following parts: high frequency inverter, energy transmitting coil, energy receiving coil, rectifier and filter circuit, impedance matching network 
and loads. Figure I is an equivalent circuit diagram for the wireless power supply system for smart home appliances.

The KVL circuit equation can be drawn accordingly:

$$
\left\{\begin{array}{l}
U_{1}=Z_{0} I_{0}-j \omega M_{1} I_{1}-j \omega M_{2} I_{2} \\
j \omega M_{i} I_{0}=Z_{i} I_{i} \quad(i=1, \ldots, N)
\end{array} .\right.
$$

In equation (1), $\mathrm{N}$ is the number of loads of smart home appliances; $Z_{0}$ is the impedance of the transmitting end and $Z_{0}=j \omega L_{0}+1 / j \omega C_{0}+R_{0}$ while $Z_{i}$ is the impedance of the receiving end and $Z_{i}=j \omega L_{i}+1 / j \omega C_{i}+R_{L i}+R_{i} ; R_{i}$ and $R_{0}$ are the internal resistance of transmitting coils and receiving coils respectively; $R_{L i}$ is the equivalent impedance of the rear end of the receiving coils, which can be adjusted by the Impedance Matching Network (IMN) on either side of loads; $L_{0}$ and $L_{i}$ are the inductance for transmitting coils and receiving coils, respectively; $C_{0}$ and $C_{i}$ are the compensation capacitance for transmitting and receiving ends, respectively [10-12].

When the loads are powered, there are certain distances among these loads. The mutual inductance among the receiving coils is neglected in this paper. When the size of the energy transmitting coils is relatively large, the mutual inductance between the transmitting coil and each receiving coil follows the rule: $M_{1}=M_{2}=\ldots=M_{i}=\ldots=M_{N}$. Then, according to equation (1), the currents in the transmitting coil and the receiving coils are

$$
\begin{aligned}
& I_{0}=\frac{U_{1}}{Z_{0}+\omega^{2} M_{1}^{2} \sum_{i=1}^{N} \frac{1}{Z_{i}}} \\
& I_{i}=\frac{j \omega M_{1} U_{1}}{Z_{i}\left(Z_{0}+\omega^{2} M_{1}^{2} \sum_{i=1}^{N} \frac{1}{Z_{i}}\right)} .
\end{aligned}
$$

Therefore, the power supply of a single load and the total loads can be expressed as

$$
\begin{aligned}
& P_{i}=I_{i}^{2} R_{L i}=\frac{\omega^{2} M_{1}^{2} U_{1}^{2} R_{L i}}{Z_{i}^{2}\left(Z_{0}+\omega^{2} M_{1}^{2} \sum_{i=1}^{N} 1 / Z_{i}\right)^{2}} . \\
& P_{o}=N P_{i}=\frac{N \omega^{2} M_{1}^{2} U_{1}^{2} R_{L i}}{Z_{i}^{2}\left(Z_{0}+\omega^{2} M_{1}^{2} \sum_{i=1}^{N} 1 / Z_{i}\right)^{2}} .
\end{aligned}
$$

According to formula (3) the transmitting efficiency of the system is

$$
\eta=\frac{\sum_{i=1}^{N} P_{i}}{U_{1} I_{0}}=\frac{\omega^{2} M_{1}^{2}}{Z_{0}+\omega^{2} M_{1}^{2} \sum_{i=1}^{N} \frac{1}{Z_{i}}} \sum_{i=1}^{N} \frac{R_{L i}}{Z_{i}^{2}} .
$$

In order to simplify the research on power control and efficiency optimization of the multi-load wireless power supply system for smart home appliances and to make it easier to design and control such a system, and also with the feasibility of the system taken into account, the receiving coils can be designed with same specifications and the equivalent impedance of the rear end of the receiving coils can be adjusted to the same by the impedance matching network. These are two important prerequisites for the theoretical analysis in the paper. Based on that, $L_{1}=L_{i}, C_{1}=C_{i}, R_{i}=R_{1}, R_{L i}=R_{L}, Z_{1}=Z_{i}$ $(i=1,2, \ldots, N)$.

By adjusting the power frequency and the size of the compensation capacitor to ensure $\omega L_{0}=1 / \omega C_{0}$ and $\omega L_{i}=1 / \omega C_{i}$ when the system can operate in a resonant state, the equivalent impedance of the transmitting and receiving end can be expressed as $Z_{0}=R_{0} \quad, \quad Z_{i}=R_{L i}+R_{i} \quad(i=1,2, \ldots, N)$, respectively. Then equation (3) and (4) can be simplified as

$$
P_{o}=\frac{N \omega^{2} M_{1}^{2} U_{1}^{2} R_{L}}{\left(R_{0}\left(R_{1}+R_{L}\right)+N \omega^{2} M_{1}^{2}\right)^{2}},
$$

$$
\eta=\frac{N \omega^{2} M_{1}^{2} R_{L}}{\left(R_{1}+R_{L}\right)\left(R_{0}\left(R_{1}+R_{L}\right)+N \omega^{2} M_{1}^{2}\right)} .
$$

\section{B. Analysis on Load Characteristics of the Wireless Power Supply System for Smart Home Appliances}

In real life, the parameters of energy transmitting and receiving coils in the wireless power supply system for smart home appliances are generally fixed, so the mutual inductance between the energy transmitting and receiving coils is also basically unchanged. In the following, the influence of the equivalent impedance of the rear end of the receiving coils on the output power and energy transfer efficiency will be studied.

The numerator and denominator of equation (5) can be multiplied by $1 / R_{L}$ simultaneously, then we get

$$
P_{o}=\frac{N \omega^{2} M_{1}^{2} U_{1}^{2}}{\frac{\left(N \omega^{2} M_{1}^{2}+R_{0} R_{1}\right)^{2}}{R_{L}}+R_{L} R_{0}^{2}+2 R_{0}\left(N \omega^{2} M_{1}^{2}+R_{0} R_{1}\right)}
$$


When $\frac{\left(N \omega^{2} M_{1}^{2}+R_{0} R_{1}\right)^{2}}{R_{L}}=R_{L} R_{0}^{2}$, namely $R_{L}=R_{L O 1}=$ $\frac{N \omega^{2} M_{1}^{2}+R_{0} R_{1}}{R_{0}}$, the output power of the system reaches its maximum value of

$$
P_{o, \max }=\frac{N \omega^{2} M_{1}^{2} U_{1}^{2}}{4 R_{0}\left(N \omega^{2} M_{1}^{2}+R_{0} R_{1}\right)}
$$

The numerator and denominator of equation (6) can be multiplied by $1 / R_{L}$ simultaneously, then we get

$$
\eta=\frac{N \omega^{2} M_{1}^{2}}{\frac{N \omega^{2} M_{1}^{2} R_{1}+R_{0} R_{1}^{2}}{R_{L}}+R_{0} R_{L}+2 R_{0} R_{1}+N \omega^{2} M_{1}^{2}}
$$

When $\frac{N \omega^{2} M_{1}^{2} R_{1}+R_{0} R_{1}^{2}}{R_{L}}=R_{0} R_{L}$, namely $R_{L}=R_{L O 2}=$ $\sqrt{\frac{R_{1}}{R_{0}}\left(N \omega^{2} M_{1}^{2}+R_{0} R_{1}\right)}$, the energy transmitting efficiency of the system reaches its maximum value of

$$
\eta_{\max }=\frac{N \omega^{2} M_{1}^{2}}{\left(\sqrt{R_{0} R_{1}+N \omega^{2} M_{1}^{2}}+\sqrt{R_{0} R_{1}}\right)^{2}} .
$$

The quality factors of the transmitting and the receiving coils are defined as $Q_{0}=\frac{\omega L_{0}}{R_{0}}, Q_{1}=\frac{\omega L_{1}}{R_{1}}$ respectively, then equation (8) and equation (9) can be further expressed as

$$
\begin{aligned}
& P_{o, \text { max }}=\frac{U_{1}^{2} N k^{2} Q_{0} Q_{1}}{4 R_{0}\left(1+N k^{2} Q_{0} Q_{1}\right)}, \\
& \eta_{\text {max }}=\frac{N k^{2} Q_{0} Q_{1}}{\left(\sqrt{1+N k^{2} Q_{0} Q_{1}}+1\right)^{2}} .
\end{aligned}
$$

Then, let $F=N k^{2} Q_{0} Q_{1}$, the maximum power and maximum efficiency values can also be approximated as

$$
P_{o, \max }=\frac{F U_{1}^{2}}{4 R_{0}(1+F)}=\left\{\begin{array}{l}
\frac{U_{1}^{2}}{4 R_{0}} \quad F>>1 \\
\frac{U_{1}^{2}}{4 R_{0}}\left(1-\frac{1}{1+F}\right)
\end{array},\right.
$$

$$
\eta_{\max }=1-\frac{2}{\sqrt{1+F}+1}
$$

Figure II depicts the relationship between the maximum output power, the maximum transmitting efficiency and $F$. As can be seen from the figure: as $F$ increases, the maximum output power will increase and then tend to reach a stable value: $U_{1}^{2} / 4 R_{0}$. The maximum transmitting efficiency will also increase correspondingly, then gradually approach $100 \%$.

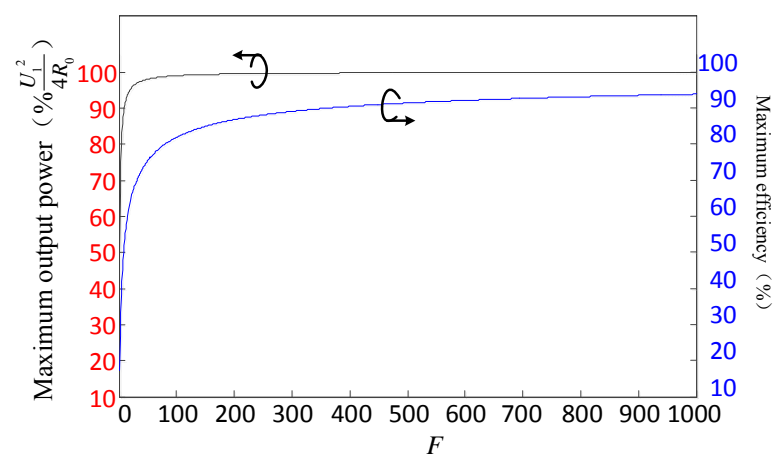

FIGURE II. THE RELATIONSHIP BETWEEN MAXIMUM POWER OUTPUT, MAXIMUM TRANSFER EFFICIENCY AND F

The above analysis shows that, in order to improve the maximum output power and maximum transmitting efficiency of the system, two measures can be taken:

1) Increase the coupling coefficient of coils. The tightness of the coupling between the coils is mainly determined by the size and structure of the coils. Under normal circumstances, the relative position of the energy dispatch coils also affects the coupling coefficient. In wireless power supply system for smart home appliances, the actual position of the load and the horizontal and vertical offset of transmitting coils are main factors that cause the coupling coefficient to decrease. In order to solve this problem, we can study energy dispatch coils with high capacity deviation ratio.

2) Design coils with high quality factor. The design of coils with high quality factor can effectively reduce the sensitivity of the system efficiency to the coupling coefficient. In practical applications, wires with low resistivity such as Litz wire can be used for coil winding. The high frequency of the system is also a trend. Note that, because of that, the overall design of the system will be more complex, and harsher electromagnetic environment will also be triggered. The quality factor of the coils should not be too high as well [13], since the offset of other parameters of the system will lead to huge fluctuations in the system when it is operating.

In addition, it can be found that $R_{L O 1}$, the load value corresponding to the maximum output power is different from $R_{\mathrm{LO} 2}$, the load value corresponding to the maximum energy transmitting efficiency, and the relationship between the two can be expressed as 


$$
\frac{R_{L O 1}}{R_{L O 2}}=\sqrt{1+F} .
$$

It is obvious that $R_{\mathrm{LO} 1}>R_{\mathrm{LO} 2}$, and the smaller $F$ is, the closer $R_{\mathrm{LO} 1}$ and $R_{\mathrm{LO} 2}$ will be. To meet $R_{\mathrm{LO} 1}=R_{\mathrm{LO} 2}$, we must ensure that $F=0$, but under such circumstances, the output power and transmitting efficiency will also be 0 , which is obviously of no meaning, so the maximum power and the maximum efficiency of the system cannot be achieved simultaneously. That means we have to strike a balance between the two according to which objective we give priority to control.

\section{CONTROL Strategies FOR PERFORMANCE OPTIMIZATION OF WIRELESS CHARGING SYSTEM FOR SMART HOME APPLIANCES}

\section{A. Power Stability Control}

Under the premise of constant output voltage of the power supply, as the numbers of loads increases, the change of the current at the receiving ends and the power supply of each single load can be seen in Figure III. As it can be seen, as the number of loads increases, the current at the transmitting end and the receiving ends will decline, which will lead to lack of power supply; as the number of loads decreases, the current at the transmitting end and the receiving ends will increase significantly, which may cause destructive damage to the system. Although using components with higher current rating can alleviate this problem, more system loss cannot be avoided. Therefore, to promote the "one-to-many" wireless power supply system for smart home appliances, the problem of instability caused by the varied number of loads to the system work must be addressed.

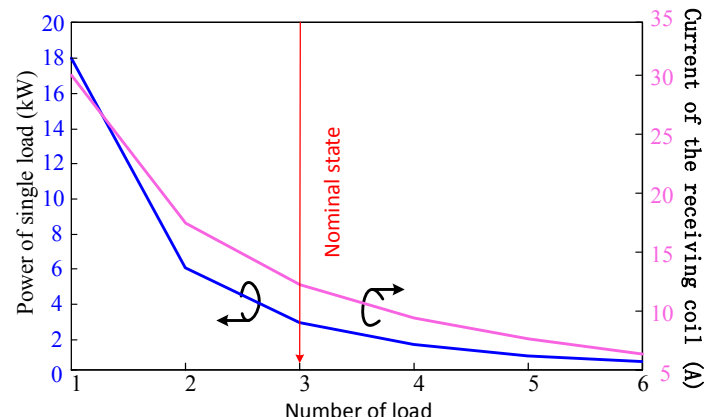

FIGURE III

RELATIONSHIP BETWEEN LOAD POWER AND RECEIVER CURRENT OF SINGLE LOAD

In order to solve the above problem and ensure the stability of power supply to the loads and the working state of the system, the power supply voltage needs to be controlled in real time according to the changing number of loads. A solution is to install a sensor to monitor the number of loads in real time and send the information back to the power control unit at the transmitting end. However, this solution cannot distinguish loads that have been powered from those that haven't, thus less feasible. Therefore, this paper will further research on how to control the power stability of the wireless power supply system for smart home appliances. In this paper, the number of loads can be measured by detecting the current at the transmitting end in real-time terms. Based on that, the voltage of power supply can be regulated. Ultimately, the stability of power supply to each single load can be achieved even though the number of loads continues to change. The workflow of the power stability control program is shown in Figure IV, with more specific control steps as follows:

Step 1. According to $I_{0}=U_{1 N} /\left(Z_{0}+N \omega^{2} M_{1}^{2} / Z_{1}\right)$, draw a look-up table of the current at the transmitting end, that is, the current at the transmitting end corresponding to different supply voltages and the number of loads. Here, $U_{1 N}$ refers to the supply voltage that can ensure that each single load can receive rated electric power when $\mathrm{N}$ loads are simultaneously powered. Drawing the look-up table of transmitting current in advance can avoid potential complicated calculations and reduce the time required for the control process.

Step 2. Initialize the power supply voltage: $U_{1}=U_{11}$. The purpose is to generate the current available for detection at the receiving ends. To avoid excessive current, $U_{11}$, the minimum voltage are selected.

Step 3. Detect the size of $I_{0}$, the current at the transmitting end.

Step 4. Identify the number of loads by referring to the look-up table according to the size of the current at the transmitting end.

Step 5. Set the voltage of power supply according to the number of loads to ensure stable power supply to each single load.

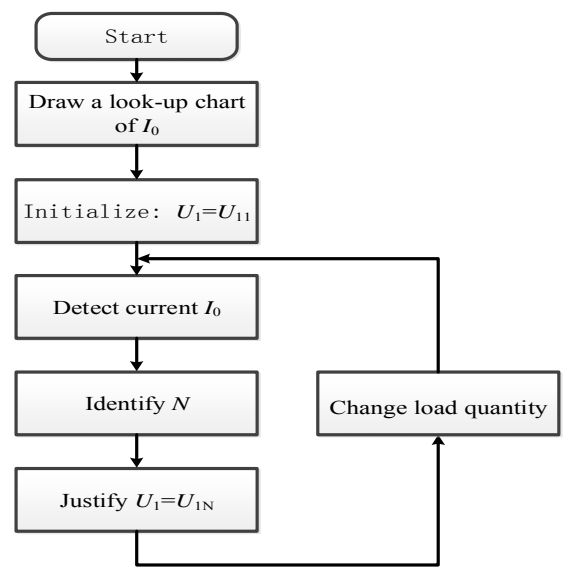

FIGURE IV.

\section{FLOW CHART OF CONTROL STRATEGY OF} POWER STABILITY

\section{B. Emergency Power Demand Control}

When some loads need emergency power supply, the above control strategy is difficult to meet the corresponding powering demand. Therefore, based on the impedance matching network, a control strategy in times of emergency power supply is proposed in this paper. By controlling the load matching 
network at the load sides, load characteristics can be adjusted and the distribution of received power can thus be changed.

Equivalent loads of home appliances can be set at a nominal value through the impedance matching. Assuming that the power demand of the n-th load is emergent, the power supply can be expressed as

$$
P_{n}=\frac{\omega^{2} M_{1}^{2} U_{1}^{2} R_{L n}}{\left(R_{L n}+R_{1}\right)^{2}\left(R_{0}+\omega^{2} M_{1}^{2} \sum_{i=1}^{N} 1 /\left(R_{L i}+R_{1}\right)\right)^{2}} .
$$

At this point, the power supply of loads with normal power demand can be expressed as

$$
P_{m}=\frac{\omega^{2} M_{1}^{2} U_{1}^{2} R_{L}}{\left(R_{L}+R_{1}\right)^{2}\left(R_{0}+\omega^{2} M_{1}^{2} \sum_{i=1}^{N} 1 /\left(R_{L i}+R_{1}\right)\right)^{2}} .
$$

Let $D=R_{0}+\omega^{2} M_{1}^{2} \sum_{i=1, i \neq n}^{N} 1 /\left(R_{L i}+R_{1}\right)$, then (16) can be simplified as

$$
\begin{aligned}
& P_{n}=\frac{\omega^{2} M_{1}{ }^{2} U_{1}{ }^{2} R_{L n}}{\left(R_{L n}+R_{1}\right)^{2}\left(D+\frac{\omega^{2} M_{1}{ }^{2}}{R_{L n}+R_{1}}\right)^{2}} \\
& =\frac{\omega^{2} M_{1}{ }^{2} U_{1}{ }^{2}}{\frac{\left(\omega^{2} M_{1}{ }^{2}+R_{1} D\right)^{2}}{R_{L n}}+R_{L n} D^{2}+2 R_{1} D^{2}+2 D \omega^{2} M_{1}{ }^{2}}
\end{aligned}
$$

From the above equation, it can be obtained that: When the equivalent loads of other loads stay unchanged, $P_{n}$, the power supply of the n-th load increases first and then decreases as $R_{L n}$ increases, and reaches its maximum value when $R_{L n, o}=\left(\omega^{2} M_{1}^{2}+R_{1} D\right) / D$ :

$$
P_{n, \max }=\frac{\omega^{2} M_{1}^{2} U_{1}^{2}}{4 D\left(R_{1} D+\omega^{2} M_{1}^{2}\right)}
$$

Therefore, when individual loads are in urgent need of power supply, the characteristics of other loads can stay unchanged and more energy can be absorbed by optimizing characteristics of the former loads. In addition, (17) can be simplified as

$$
P_{m}=\frac{\omega^{2} M_{1}^{2} U_{1}^{2} R_{L}}{\left(\left(R_{L}+R_{1}\right) D+\frac{\omega^{2} M_{1}^{2}\left(R_{L}+R_{1}\right)}{R_{L n}+R_{1}}\right)^{2}} .
$$

From the above equation, we can see that: the power supply to loads with normal power demand will increase as the variable load $R_{L n}$ increases, which means that to meet the emergency power demand of certain loads, the cost is power supply fluctuations suffered by other loads, so this control strategy is only applicable to the case of emergency power supply demand.

\section{EXPERIMENTAL RESEARCH ON THE WIRELESS POWER SUPPLY SYSTEM FOR SMART HOME APPLIANCES}

\section{A. The Overall Design of the System}

In order to verify the characteristics of the loads in the system and the control strategy of power supply, a small-scale experimental platform was set up, as shown in Figure V. The current generated by the direct current source is converted by a high frequency inverter into an alternating current of $85 \mathrm{kHz}$ and transmitted to the receiving ends through the electromagnetic coupling between the energy transmitting coil and the energy receiving coils. The receiving coils directly supply power to the resistive loads.

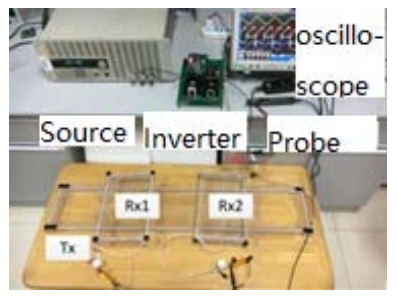

FIGURE V.

EXPERIMENT PLATFORM OF WIRELESS POWER SUPPLY SYSTEM OF MULTIPLE LOAD

Transmitting and receiving coils are made from 2025 strands of Litz wires, each of which has a diameter of $0.05 \mathrm{~mm}$. $\mathrm{Rx} 1, \mathrm{Rx} 2$ stand for the spiral rectangular coil, with more specific parameters shown in Table 1. The transmission distance between the transmitting and the receiving coils is $5 \mathrm{~cm}$.

TABLE I. PARAMETERS OF ENERGY SENDING AND RECEIVING COIL

\begin{tabular}{|c|c|c|c|c|}
\hline Coil & Size(m) & Resistance $(\Omega)$ & Inductance $(\boldsymbol{\mu H})$ & Capacitance $(\boldsymbol{n} \boldsymbol{F})$ \\
\hline $\mathrm{Tx}$ & $1.00 \times 0.20$ & 0.080 & 71.2 & 49 \\
\hline $\mathrm{R} x 1$ & $0.30 \times 0.20$ & 0.030 & 25.8 & 136 \\
\hline $\mathrm{Rx} 2$ & $0.30 \times 0.20$ & 0.030 & 25.9 & 136 \\
\hline
\end{tabular}

B. Experiment on the Influence of Loads on Power Supply and Transmitting Efficiency

The above analysis shows that the number of loads with power supply and their equivalent impedances have a great impact on the power supply and transmitting efficiency. In this paper, power resistances are used to represent loads, and the fixed input voltage is $17.0 \mathrm{~V}$. The output power of the system is measured with the change of load resistances when $\mathrm{N}$ (the number of power loads) equals to 1,2 , and 3 . The experimental results and Matlab results are shown in Figure VI (the curves in the figure below represent the results of Matlab calculation, and 
the dots represent the experimental test results). As can be seen from the figure, with the increase of the load resistance, the total power supply of the system increases first and then decreases gradually. There is an optimal load resistance value that maximizes the total power supply of the system and the corresponding optimal load value are $2 \Omega, 5 \Omega$ and $7 \Omega$, respectively. The maximum output power increases with the increase of the number of loads with power supply, which is consistent with the theoretical analysis.

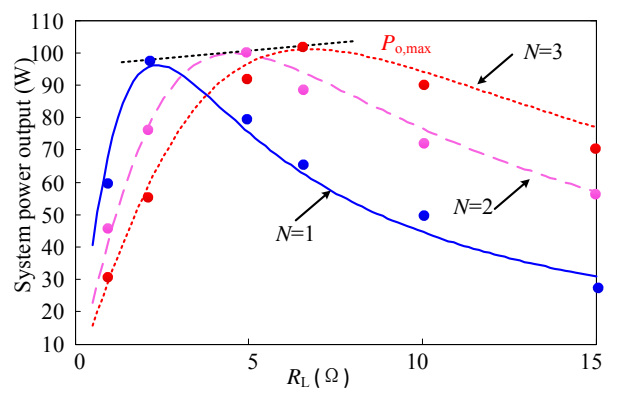

FIGURE VI. CHANGE OF SUPPLY POWER WITH LOAD

By measuring the input power of the system when $\mathrm{N}$ (the number of loads with power supply) equals to 1,2 , and 3, respectively, the change of the transmitting efficiency of the system is shown in Figure VII as the load resistance changes. As can be seen from the figure, the greater the number of loads is, the higher the transmitting efficiency of the system will be, which is consistent with the theoretical analysis. For a certain number of loads, the system transmitting efficiency increases first and then decreases as load resistance increases, and there is an optimal load resistance value that maximizes the system efficiency, with the corresponding optimal load value being 1 $\Omega, 1.5 \Omega$ and $2 \Omega$. The maximum transmitting efficiency increases as the number of loads grows, which is due to the increased mutual inductance.

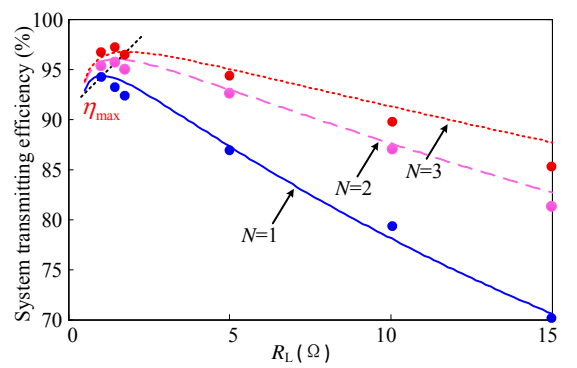

\section{FIGURE VII. VARIATION OF TRANSFER EFFICIENCY WITH LOAD}

When compared with the results calculated by theoretical formulas, the experimental values show a certain deviation. This is because the actual values of the inductance and the capacitance cannot reach the full resonance state. However, both the experimental results and the theoretical analysis show the same variation rule. In summary, the following conclusions can be drawn: 1) There is an optimal load value that maximizes the output power of the system and the maximum output power of the system remains unchanged even with different loads; 2) There is an optimal load value that maximizes the transmitting efficiency of the system. As the number of loads increases, the maximum transmitting efficiency of the system will also increase, which is due to the improvement of the overall coupling coefficient. 3) The optimal load values corresponding to the maximum output power and the maximum transmitting efficiency are different.

\section{Experiment on Power Supply Control}

In order to avoid fluctuating power supply caused by the change of load quantity, this paper measures the change of current at the transmitting end when the number of load is changing. The rated supply power of the resistor load (with the resistance of $4 \Omega$ ) is $50 \mathrm{~W}$. The rated current and the current at the transmitting end are $3.5 \mathrm{~A}$ and $8.3 \mathrm{~A}$, respectively after calculation. Table 2 is a look-up table recording the current at the transmitting end gained from the above theoretical analysis of the system. The number of loads with power supply ranges from 1 to 4 , with the corresponding rated input voltage being $13.0 \mathrm{~V}, 17.0 \mathrm{~V}$ and $22.0 \mathrm{~V}$, respectively. When the supply voltage is fixed, the quantity change of loads with power supply can be reflected in the change of the current at the transmitting end.

\section{TABLE II. TAB.2 LOOK-UP TABLE OF CURRENT}

\begin{tabular}{|c|c|c|c|c|}
\hline $\mathrm{J}_{\mathrm{N}}(\mathrm{V})$ & 1 & 2 & 3 & 4 \\
\hline 13.0 & 8.3 & 6.4 & 4.9 & 4.0 \\
\hline 17.0 & 10.9 & 8.3 & 6.5 & 5.2 \\
\hline 22.0 & 14.1 & 10.8 & 8.3 & 6.7 \\
\hline
\end{tabular}

When the input voltage is fixed at $17.0 \mathrm{~V}$, as the number of loads with power supply changes, the change of the currents at the transmitting and receiving end is shown in Figure VIII. It can be seen that the currents at the transmitting and receiving ends will increase as the number of loads decreases. For example, when the number of loads changes from 2 to 1 , by adjusting the input voltage of the power supply according to the current look-up table, the change of the current at the transmitting end is shown in Figure IX. When the number of loads changes abruptly, the effective value of the emitter current changes from $8.14 \mathrm{~A}$ to $10.8 \mathrm{~A}$, then after adjustment, the value returns to $8.14 \mathrm{~A}$. The power supply is also stabilized.

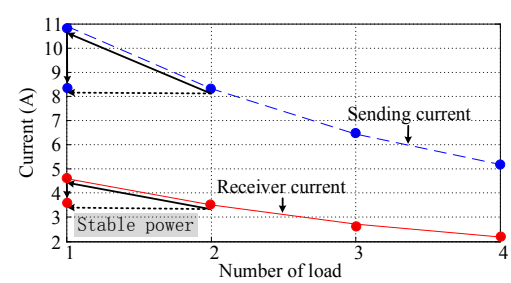

FIGURE VIII. INFLUENCE OF NUMBER OF LOAD ON SENDING AND RECEIVING CURRENT 


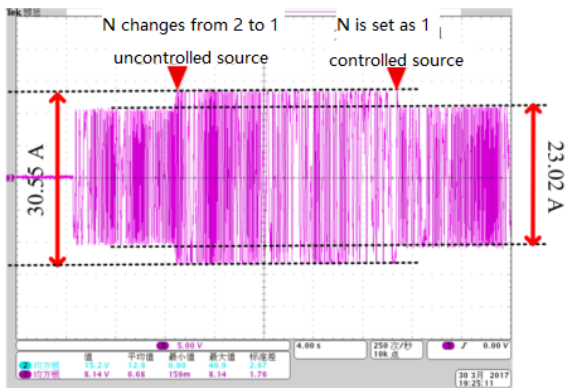

FIGURE IX.

CURRENT CHANGE OF SENDING COIL

When the input voltage is fixed at $17.0 \mathrm{~V}$, take the case when $\mathrm{N}=2, \mathrm{~N}=3$, and $\mathrm{N}=4$, by changing the values of load resistances with emergent power demand and keeping the resistance of other loads unchanged, the relationship between power distribution and the load resistance with emergent power demand is shown in Figure X. Here, the working point when all the loads have the same resistance is defined as the average power point, as shown in Figure $\mathrm{X}$, point 1, and the working point when the loads with emergent power demand reach their maximum receiving power is defined as the maximum receiving power point, as shown in Figure X, point 2 .

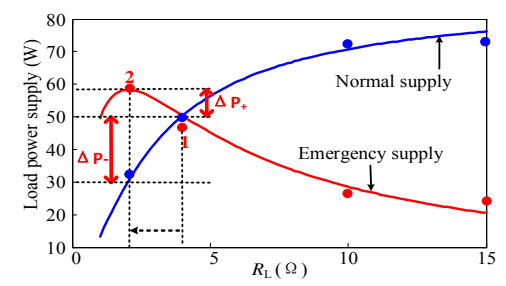

(a) $\mathrm{N}=2$

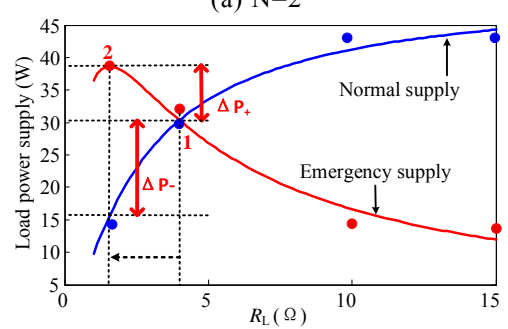

(b) $\mathrm{N}=3$

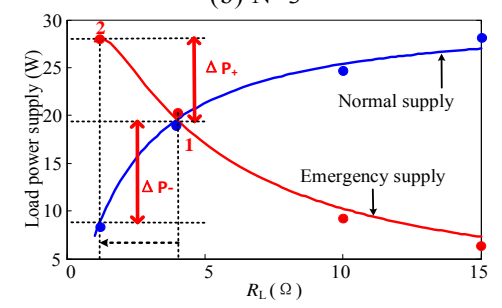

(c) $\mathrm{N}=4$

FIGURE X. INFLUENCE OF EMERGENCY POWER DEMAND LOAD ON POWER DISTRIBUTION

When $\mathrm{N}=2$, by adjusting the resistance of loads with emergent power demand along the direction of the dotted arrow in the figure, and shifting the operating point from point 1 to point 2 , the power supply to the loads with emergent power demand increases from $50 \mathrm{~W}$ to $58 \mathrm{~W}$, while the power supply to other loads decreases from $50 \mathrm{~W}$ to $30 \mathrm{~W}$. When $\mathrm{N}=3$, by adjusting the resistance of loads with emergent power demand along the direction of the dotted arrow in the figure, and shifting the operating point from point 1 to point 2 , the power supply to the loads with emergent power demand increases from $30 \mathrm{~W}$ to $40 \mathrm{~W}$, while the power supply to other loads decreases from $30 \mathrm{~W}$ to $15 \mathrm{~W}$. When $\mathrm{N}=3$, by adjusting the resistance of loads with emergent power demand along the direction of the dotted arrow in the figure, and shifting the operating point from point 1 to point 2 , the power supply to the loads with emergent power demand increases from $20 \mathrm{~W}$ to $28 \mathrm{~W}$, while the power supply to other loads decreases from $20 \mathrm{~W}$ to $9 \mathrm{~W}$. when we define the increased power of loads with emergent power demand as $\Delta \mathrm{P}+$, the reduced power of other fixed loads as $\Delta \mathrm{P}$-, when $\mathrm{N}=2, \mathrm{~N}=3$ and $\mathrm{N}=4$, the corresponding $\Delta \mathrm{P}+/ \Delta \mathrm{P}$ - is $0.4,0.66$ and 0.73 , respectively. The greater the number of loads with power supply is, the less impact will be exerted on other fixed loads, and the more significant the effect of such power regulation means is.

\section{CONCLUSIONS}

This paper studies the working mechanism of the wireless power supply system for smart home appliances and the control strategies for performance optimization. The structure of the wireless power supply system for smart home appliances is introduced and the equivalent circuit model of "one-to-many" wireless power supply system for smart home appliances is established. On this basis, the load characteristics of the system are analyzed. The paper also studies how the number of loads will influence the charging power and transmitting efficiency of the system. An optimal load value, which can ensure the maximum of the charging power and maximum transmitting efficiency, was found. As for the instability of power supply caused by the change of load quantity in the wireless power supply system, a power stability control strategy based on current detection of the transmitting end is proposed. In the light of the situation when some loads require emergent power supply, a control strategy is also suggested. A hardware platform for the system was built and performance optimization experiment was carried out to verify the correctness of the theoretical derivation.

\section{ACKNOWLEDGEMENT}

This research is supported by China Southern Power Grid Corporation (Research and Application of Key Technologies of Intelligent Cloud Service in Hainan Power Grid).

\section{REFERENCES}

[1] K Feng, S Tong, "Origin and trend of development of smart home appliances," in China New Technologies and Products, vol. 23, no. 6, pp. $1-7,2010$.

[2] G Wang. "Practice and Prospect of China intelligent power utilization," in Electric Power, vol. 45, no. 1, pp. 1-5, 2012.

[3] R Abhishek, K D B Soumya, B Amiya, et al. "Location Aware Resource Management in Smart Homes," Proceedings of the First IEEE International Conference on Pervasive Computing and Communications (PerCom'03). IEEE CS Press, 2003.

[4] Q Wang, Y Zhu, G Ge, et al. "Application discussion of packet transfer technology for smart power distribution and utilization networks," in Power System Protection and Control, vol. 42, no. 9, pp. 139-146, 2014. 
[5] Q Tang, N Liu, J Zhang. "Theory and key problems for automated demand response of user side considering generalized demand side resources," in Power System Protection and Control, vol. 42, no. 24, pp. 138-147, 2014.

[6] X Huang, L Tan, Z Chen, et al. "Review and research progress of wireless power transfer technology," in Transactions of China Electrotechnical Society, vol. 28, no. 10, pp. 1-11, 2013.

[7] S Cheng, X Chen, J Wang, et al. "Key technologies and applications of wireless power transmission," in Transactions of China Electrotechnical Society, vol. 30, no. 19, pp. 68-84, 2015.

[8] M Soljačić, A Kurs, A Karalis, et al. "Wireless power transfer via strongly coupled magnetic resonances," in Science Express, vol. 112, no. 6, pp. 1-10, 2007.

[9] X Zhang, F Zhou. "Smart grid leads the journey to innovative smart home and energy consumption patterns," in Power System Protection and Control, vol. 42, no. 5, pp. 59-67, 2014.

[10] B X Nguyen, D M Vilathgamuwa, G H B Foo, et al. "An Efficiency Optimization Scheme for Bidirectional Inductive Power Transfer Systems," in IEEE Transactions on Power Electronics, vol. 30, no. 11, pp. 6310-6319, 2015.

[11] W Fu, B Zhang, D Qiu, et al. "Maximum efficiency analysis and design of self-resonance coupling coils for wireless power transmission system," in Proceedings of the CSEE, vol. 29, no. 18, pp. 21-26, 2009.

[12] R L Steigerwald. "A comparison of half-bridge resonant converter topologies," in IEEE Transactions on Power Electronics, vol. 3, no. 2, pp. 174-182, 2002.

[13] Z Pantic, S M Lukic. "Framework and Topology for Active Tuning of Parallel Compensated Receivers in Power Transfer Systems," in IEEE Transactions on Power Electronics, vol. 27, no. 11, pp. 4503-4513, 2012. 